\title{
On Massless Fields with Arbitrary Spin
}

R. ILLGE

Herrn Prof. Dr. V. Wünsch zum 50. Geburtstag hochachtungsvoll gewidmet

A gauge field theory is proposed for symmetric spinor fields of valence $(n+1,0)$ on a flat spacetime which generalizes the Maxwell theory of electromagnetism. Further we study first. order equations for symmetric spinor fields of valence $(1, n)$ which are consistent in arbitrarily curved spacetimes, conformally and gauge invariant and whose solutions satisf $y$ the equations for the potentials of massless fields in flat spacetimes.

Key words: higher spin, gauge field theory, curved spacetimes

AMS subject classification: $81 \mathrm{D} 25,81 \mathrm{E} 20,83 \mathrm{C} 60$

\section{Introduction}

It is well known $[3,8]$ that the (massless or massive) field equations proposed by P.A.M. DIRAC [5] and M. FIERZ [7] become inconsistent if the spin is greater than one and an electromagnetic or gravitational field is present. The zero rest-mass equations

$$
\nabla_{X}^{A} \varphi_{A A_{1}} \ldots A_{n}=0
$$

are consistent for $n=0$ (Weyl's equation) and $n=1$. (homogeneous Maxwell's equation), but for $n>1$ they are inconsistent unless the spacetime is conformally flat $[3,13,15]$. In complexified spacetimes the equations. (1) are consistent for $n>1$ only if the spacetime is conformally self-dual [13,14]. In Riemann-Cartan spacetimes there are also consistency conditions which restrict the "background geometry". [1].

There are several attempts to overcome these difficulties (see, e.g., [2]). The theory of M. FIERz and W. PAULI [8] which is based on an action principle yields consistent field equations for arbitrary spin, but it is necessary to introduce certain auxiliary fields in order to have enough field components 
(see also $[6,9]$ ). Simple supergravity is a consistent field theory to describe the interaction of a massless spin $3 / 2$-field with a gravitational field, but supergravity seems to be unsuitable to describe fields of arbitrary spin [11]. All of these theories are not suitable to describe massless fields with arbitrary spin on a generally curved spacetime.

In the first part of this paper we consider massless fields in flat spacetimes and generalize the well known relations between an electromagnetic field and its potential to fields of higher spins. It is shown that for every symmetric spinor field $\varphi$ the equation

$$
\varphi_{A A_{1} \ldots A_{n}}=\partial_{\left(A_{1}\right.}^{\dot{x}_{1}} \ldots \partial_{A_{n}}^{\dot{X}_{n}} \xi_{A) \dot{x}_{1}} \ldots \dot{x}_{n}
$$

has a solution $\xi$ which is symmetric in the dotted indices. The potential $\xi$ of the field $\varphi$ is not uniquely determined by (2) but there is a gauge freedom as in electromagnetic theory. If $\varphi$ satisfies the field equation (1), then each potential $\xi$ belonging to $\varphi$ according to (2) satisfies the field equation

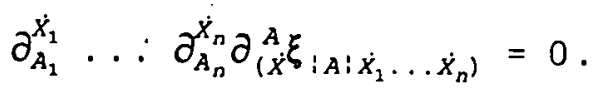

It seems to be impossible to generalize this theory to curved spacetimes. But the equations

$$
\nabla_{\left(\dot{X} \dot{X}_{i}: \dot{X}_{1} \ldots \dot{X}_{n}\right)}^{A}=0
$$

which are obviously stronger than (3) can be solved in arbitrarily curved spacetimes for arbitrary $n$. They have some remarkable properties (cf. Theorem 1):

i) The equations (4) are consistent in arbitrarily curved spacetimes. The Cauchy problem is properly posed for these equations.

ii) The equations (4) are conformally invariant.

iii) If 0 is a symmetric spinor field.with $n-1$ dotted indices, then there exists a solution $\xi$ of (4) such that

$$
\sigma^{c z} \xi_{C \dot{X}_{1} \ldots \dot{x}_{n-1} \dot{z}}=\tilde{\omega}_{\dot{X}_{1} \ldots \dot{X}_{n-1}} \text {. }
$$

These results show that (4) is a suitable candidate to describe 
(possibly a special class of) massless spinor fields with arbitrary spin.

In the following, we assume that the (four-dimensional) spacetime and all given spinor fields are of class $C^{*}$. We denote by $\hat{s}_{n, k}(n, k=0,1,2, \ldots)$ the set of all symmetric spinor fields with $n$ undotted and $k$ dotted indices:

$$
\eta \in \hat{S}_{n, k} \Leftrightarrow \eta_{A_{1} \ldots A_{n} \dot{x}_{1} \ldots \dot{x}_{k}}=\eta_{\left(A_{1} \ldots A_{n}\right)\left(\dot{x}_{1} \ldots \dot{x}_{k}\right)}
$$

Further we use the notation of [13]. Especially, $\Psi \in \hat{S}_{4,0}$ denotes the Weyl spinor and $2 \Phi \in \hat{S}_{2,2}$ the spinor equivalent of the tracefree part of the Ricci tensor.

\section{Spinor fields on flat spacetimes}

The first lemma is used to prove the existence of a potential for a given symmetric spinor field.

Lemma 1: Let $\eta \in \hat{S}_{n+1, k}(k \geq 1)$ be some given spinor field which is divergence-free:

$$
\partial^{A \dot{x}_{1}} \eta_{A A_{1} \ldots A_{n} \dot{x}_{1} \ldots \dot{x}_{k}}=0
$$

Then the equation

$$
\partial_{A}^{\dot{z}} \dot{\mathrm{O}}_{A_{1} \ldots A_{n} \dot{z} \dot{x}_{1} \ldots \dot{x}_{k}}=\eta_{A A_{1} \ldots A_{n} \dot{x}_{1} \ldots \dot{x}_{k}}
$$

has a solution $\delta \in \hat{S}_{n, k+1}$ which is divergence-free, too.

Proof: We prove Lemma, 1 only for $n=0, k=1$, because we may append any number of free indices simultaneously on $\eta$ and $\theta$ in flat spacetimes (see, e.g., [4]). If any solution of (6) does exist, then the vanishing of its divergence follows immediately from (6).

For $n=0, k=1$ the tensor equivalent of the spinor field $\eta$ is a complex vector field. If we denote its real and imaginary part by $v$ resp. $w$, then the condition (5) yields

$$
\partial^{a} v_{a}=\partial^{a} w_{a}=0 \text {. }
$$


By the Lemma of Poincaré there exists an alternating 2-tensor $E$ with

$$
w_{a}=-\frac{1}{3} \partial^{c *} E_{c a},
$$

where the asterisk denotes the dual of $E$ [13].

with the tensor fields $E$ and $v$ let us form the equation

$$
\partial^{c} \partial_{[c} u_{a]} \equiv \frac{1}{2}\left(\partial^{c} \partial_{c} u_{a}-\partial^{c} \partial_{a} u_{c}\right)=v_{a}-\frac{1}{3} \partial^{c} E_{c a}
$$

for the vector field $u$. If we take $u$ as an electromagnetic potential, then ( 9 ) is just an inhomogeneous Maxwell's equation. The first part of (7) guarantees that the right-hand side of (9) satisfies the continuity equation. If the Lorentz gauge condition holds, then ( 9 ) reduces to an equation of normal hyperbolic type and can be solved in the usual way.

Let us now define the antisymetric tensor field $F$ by

$$
F_{a b}=\frac{2}{3} E_{a b}+2 \partial_{[a} u_{b]} .
$$

Using (8) and (9) we obtain

$$
\begin{gathered}
\partial^{a *} F_{a b}=\frac{2}{3} \partial^{a *} E_{a b}=-2 w_{b} \\
\partial^{a} F_{a b}=2 \partial^{a} \partial_{[a} u_{b]}+\frac{2}{3} \partial^{a} E_{a b}=2 v_{b}
\end{gathered}
$$

or together

$$
\frac{1}{2} \partial^{a}\left(F_{a b}-i^{*} F_{a b}\right)=\eta_{b}
$$

The spinor equivalent of the tensor $\frac{1}{2}\left(F_{\mathrm{ab}}-i^{*} F_{\mathrm{ab}}\right)$ has the form $\boldsymbol{b}_{\mathrm{XY}} \epsilon_{\mathrm{AB}}$ with a symmetric spinor 0 [13], hence the equation.(11) is equivalent to (6) with $n=0, k=1$ and the Lemma is proved

Proposition 1: Let $\varphi \in \hat{S}_{n+1,0}$ be some given spinor field. i) There exists a symmetric, divergence-free spinor field ${ }^{(n)} \xi \in \hat{S}_{1, n}$ such that

$$
\varphi_{A A_{1} \ldots A_{n}}=\partial_{A_{1}}^{\dot{x}_{1}} \ldots \partial_{A_{n}}^{\dot{X}_{n}(n)} \xi_{A \dot{X}_{1} \ldots \dot{X}_{n}} .
$$


ii) If $\xi \in \hat{s}_{1, n}$ is some spinor field which satisfies the equation

$$
\varphi_{A_{1} \ldots A_{n}}=\partial_{\left(A_{1}\right.}^{\dot{x}_{1}} \ldots \partial_{A_{n}}^{\dot{x}_{n}} \xi_{A) \dot{x}_{1} \ldots \dot{x}_{n}}
$$

and $\omega \in \hat{S}_{0, n-1}$, then the spinor field

$$
\xi_{A \dot{X}_{1} \ldots \dot{x}_{n}}=\xi_{A \dot{x}_{1} \ldots \dot{x}_{n}}+\partial_{A\left(\dot{x}_{1}\right.} \omega_{\left.\dot{x}_{2} \ldots \dot{x}_{n}\right)}
$$

satisfies equation (12), too.

Proof: i) Let us consider the following recursive system of differential equations for a sequence of spinor fields ${ }^{(1)} \xi \in \hat{S}_{n, 1}$, $\ldots,{ }^{(n)} \xi \in \hat{S}_{1, n}$ :

$$
\begin{aligned}
& \partial_{A_{1}}^{Z}{ }^{(1)} \xi_{A_{2} \ldots A_{n} A Z}=\varphi_{A A_{1} \ldots A_{n}} \\
& \partial_{A_{1}}^{\dot{Z}}(i) \xi_{A_{i \cdot 1} \ldots A_{n} A X_{1} \ldots X_{i-1} Z}=(i-1) \xi_{A_{i} \ldots A_{n} A \dot{X}_{1} \ldots \dot{X}_{i-1}}(i=2, \ldots, n)
\end{aligned}
$$

The first equation of (14) possesses (even on a generally curved spacetime [10]) a solution ${ }^{(1)} \xi \in \hat{S}_{n, 1}$ which is divergence-free. Then the second equation of (14) has a divergence-free solution (2) $\xi \in \hat{S}_{n-1,2}$ by Lemma 1 . By repeated use of Lemma 1 one obtains a sequence of divergence-free spinor fields ${ }^{(i)} \xi \in \hat{S}_{n-i+1, i}$ which satisfies the system (14) and the statement i) follows by insertion step by step.

ii) It suffices to show that

$$
\partial_{\left(A_{1}\right.}^{\dot{x}_{1}} \ldots \partial_{A_{n}}^{\dot{x}_{n}} \partial_{A)}\left(\dot{x}_{1} \omega_{\dot{x}_{2}} \ldots \dot{x}_{n}\right)
$$

vanishes for arbitrary $\omega \in \hat{S}_{0, n-1}$. Because one can interchange the derivatives in flat spacetimes the expression

$$
\partial \ldots \partial_{B}^{x_{i}} \ldots \partial_{C \dot{x}_{i}} \omega \ldots
$$

(irrelevant indices are suppressed) is antisymetric with reference to the indices $B C$. Therefore the symmetric part of the derivatives (15) must vanish 
Definition: Let $\varphi \in \hat{S}_{n+1,0}$ be some given spinor field. Then each spinor field $\xi \in \hat{S}_{1, n}$ satisfying (12) is called a potential of the field $\varphi$. The potential $\xi$ adrits a gauge transformation with $\omega \in \hat{S}_{0, n-1}$ according to (13).

Proposition 2: Let $\varphi \in \hat{S}_{n+1,0}$ and $w \in \hat{S}_{0, n-1}$ given spinor fields. Then there exists a potential of the field $\phi$ which satisfies the equations (12) and

$$
\partial^{A \dot{X}} \tilde{\xi}_{A \dot{X}_{1} \dot{X}_{2}} \ldots \dot{X}_{n}=\tilde{\omega}_{\dot{X}_{2} \ldots \dot{X}_{n}} .
$$

Proof: Let $\omega \in \hat{S}_{0, n-1}$ be a solution of the inhomogeneous wave equation

$$
\partial^{a} \partial_{a} \omega_{\dot{X}_{2} \ldots \dot{x}_{n}}=\frac{2 n}{n+1} \tilde{\omega}_{\dot{X}_{2}} \ldots \dot{x}_{n}
$$

and ${ }^{(n)} \xi \in \hat{S}_{1, n}$ the spinor field which exists according to Proposition $1 i$ ). Let us put

$$
\tilde{\xi}_{A \dot{X}_{1} \ldots \dot{X}_{n}}={ }^{(n)} \xi_{A \dot{x}_{1} \ldots \dot{X}_{n}}+\partial_{A\left(\dot{X}_{1}\right.} \omega_{\left.\dot{X}_{2} \ldots \dot{X}_{n}\right)} \cdot
$$

Because $^{(n)} \xi$ is divergence-free, we obtain by use of (17)

$$
\begin{aligned}
& \partial^{A \dot{x_{1}}} \tilde{\xi}_{A \dot{X}_{1} \ldots \dot{X}_{n}}=\partial^{A \dot{x_{1}}} \partial_{A\left(\dot{x}_{1}\right.} \dot{\omega}_{\left.\dot{X}_{2} \ldots \dot{x}_{n}\right)} \\
&=\frac{n+1}{2 n} \partial^{a} \partial_{a} \omega_{\dot{x}_{2} \ldots \dot{x}_{n}}=\tilde{\omega}_{\dot{X}_{2} \ldots \dot{x}_{n}}
\end{aligned}
$$

Remark 1: Proposition 1 generalizes some well known relations from the theory of electromagnetic fields. If $F_{b c}$ and $A_{c}$ are the field tensor and the vector potential, respectively, then $F_{b c}=$ $\partial_{b} A_{c}-\partial_{c} A_{b}$ is equivalent to (12) with $n=1$. The gauge freedom is given by (13) according to $A_{c} \Rightarrow \hat{A}_{c}=A_{c}+\partial_{c} \omega$ or by (16) according to $\partial^{c} \tilde{A}_{c}=\sigma$ (especially Lorentz gauge $\partial^{c} \tilde{A}_{c}=0$ ). Note that Proposition 1 is proved without any supposition like the equation $\partial_{[a} F_{b c]}=0$ for $F$ or, generally, like the equation (20) for $\varphi$ (see also $[10])$. 
Lemma 2: For every $\xi \in \hat{S}_{i, n}$ we have

$$
\partial_{\dot{X}}^{A} \partial_{\left(A_{1}\right.}^{\dot{X}_{1}} \ldots \partial_{A_{n}}^{\dot{X}_{n}} \xi_{A) \dot{X}_{1}} \ldots \dot{X}_{n}=\partial_{A_{1}}^{\dot{X}_{1}} \ldots \partial_{A_{n}}^{\dot{X}_{n}} \partial_{(\dot{X}}^{A} \xi_{\left.i A i \dot{X}_{1} \ldots \dot{X}_{n}\right)} \cdot
$$

Proof: Since the derivatives commute the expression

$$
\partial_{A_{1}}^{\dot{x}_{1}} \ldots \partial_{A_{n}}^{\dot{X}_{n}} \xi_{A \dot{X}_{1}} \ldots \dot{X}_{n}
$$

is symmetric with reference to the indices $A_{1} \ldots A_{n}$. By use of this fact, one obtains formula (19) by means of repeated interchange of the derivatives

Proposition 3: For a massless field $\varphi$ and its potential $\xi$ there holds:

i) If $\varphi \in \hat{S}_{n+1,0}$ is some spinor field which satisfies the equation

$$
\partial_{\dot{X}}^{A} \varphi_{A A_{1}} \ldots A_{n}=0
$$

then every $\xi \in \hat{S}_{1, n}$ which is related to $\phi$ by (12) satisfies the equation

$$
\partial_{A_{1}}^{\dot{x}_{1}} \ldots \partial_{A_{n}}^{\dot{X}_{n}} \partial_{(\dot{X}}^{A} \xi_{\left.i A i \dot{X}_{1} \ldots \dot{X}_{n}\right)}=0
$$

ii) If $\xi \in \hat{S}_{1, n}$ is some spinor field which satisfies the equation (21), then the spinor field $\varphi \in \hat{S}_{n+1,0}$ defined by (12) satisfies equation (20).

Proof: The statements of Proposition 3 follow immediately from Lemma 2

Froposition 3 shows an equivalence between the equation (20) for a massless field $\varphi$ and the equation (21) for its potential $\xi$. But equation $(20)$ is inconsistent for $n>1$ in curved spacetimes which are not conformally flat.

\section{Curved spacetimes}

We now try to modify the results of the preceding section for 
curved spacetimes. First we consider the cases of "lower" spin $(n=0$ and $n=1)$.

If $n=0$, then the Propositions 1 and 3 are void. In this case (20) is the Weyl equation, it is consistent in arbitrarily curved spacetimes (see [15]). For weyl fields there does not exist a potential.

For $n=1$, Proposition 1 is also true in curved spacetimes ([10], the second part is trivial because the gauge field $\omega$ is a scalar field). Lemma 2 is for $n=1$ universally valid, too:

Lemma 3: For every $\xi \in \hat{S}_{1,1}$ we have

$$
\nabla_{X}^{A} \nabla_{(B}^{\dot{z}} \xi_{A) \dot{Z}}=\nabla_{B}^{\dot{z}} \nabla_{\left(\dot{X} \xi_{i A: Z)}^{A}\right.}
$$

Proof: The validity of (22) follows from the Ricci identities for spinor fields (see [13])

From Lemma 3 we can deduce the following

Proposition 4: For a massless spin-1 field $\varphi$ and its potential $\xi$ there holds:

i) If $\varphi \in S_{2,0}$ is some spinor field which satisfies the equation

$$
\nabla_{\dot{X}}^{A} \varphi_{A B}=0
$$

then every $\boldsymbol{\xi} \in S_{1,1}$ such that

$$
\varphi_{A B}=\nabla_{(A}^{\dot{x}} \xi_{B) \dot{x}}
$$

satisfies the differential equation

$$
\nabla_{B}^{\dot{Z}} \nabla_{(\dot{X}}^{A} \xi_{i A i \dot{Z})}=0
$$

ii) If $\xi \in S_{1,1}$ is some spinor field which satisfies the equation (25), then the spinor field $\varphi \in S_{2,0}$ defined by (24) satisfies the equation (23).

Remark 2: The field equations (23) are equivalent to the homogeneous Maxwell's equations; they are consistent in arbitra- 
rily curved spacetimes (see, e.g., $[13,15])$. The gauge field $\omega$ can be choosen in such a way that the potential $\xi$ becomes divergence-free $\left(\nabla^{A x} \xi_{A x}=0\right.$, Lorentz gauge, $c f$. Proposition 2 and Remark $1)$. In this case equation (25) is equivalent to

$$
\nabla^{d} \nabla_{d} \xi_{B X}-2 \phi_{B C X \dot{Z}} \xi^{c \dot{Z}}+6 \Lambda \xi_{B X}=0
$$

which we obtain by use of the Ricci identities. The tensor form of this equation is just

$$
\nabla^{d} \nabla_{d} \xi_{a}-R_{a d} \xi^{d}=0
$$

which is the well-known equation for the electromagnetic potential. Therefore we can take Proposition 4 as a generalization of Maxwell's equations for complex vector potentials.

For $n>1$ the field equations (20) are inconsistent unless the spacetime is conformally flat $[3,15]$. The equations (6) are inconsistent for $n>0, k>1$ unless the spacetime has constant curvature $[10,15]$. In general, the derivative

$$
\nabla_{A_{1}}^{\dot{x}_{1}} \nabla_{A_{2}}^{\dot{x}_{2}} \xi_{A \dot{x_{1}} \dot{x}_{2}}
$$

for some spinor field $\xi \in \hat{S}_{1,2}$ is not symmetric with reference to the indices $A_{1} A_{2}$. Furthermore we obtain after a long calculation by use of the Ricci identities

$$
\begin{aligned}
& \nabla_{\dot{X}}^{A} \nabla_{\left(A_{1}\right.}^{\dot{X}_{1}} \nabla_{A_{2}}^{\dot{X}_{2}} \xi_{A) \dot{X}_{1} \dot{X_{2}}}-\nabla_{\left(A_{1}\right.}^{\dot{X}_{1}} \nabla_{\left.A_{2}\right)}^{\dot{X}_{2}} \nabla_{(\dot{X}}^{A} \xi_{\left.: A: \dot{X_{2}} \dot{X}_{2}\right)} \\
& =\Psi_{A_{1} A_{2} C_{1} C_{2}} \nabla_{\dot{Z}}^{C_{1}} \xi_{\dot{X}}^{C_{2} \dot{z}}+\bar{\Psi}_{X Z_{1} z_{2} z_{3}} \nabla_{\left(A_{1}\right.}^{\dot{z}_{1}} \xi_{\left.A_{2}\right)}^{\dot{Z}_{2} \dot{z}_{3}} \\
& -\xi^{C_{1} \dot{z}}{ }_{\dot{x}} \nabla_{\dot{z}}^{\mathcal{C}_{2}} \Psi_{A_{1} A_{2} C_{2} C_{2}}+\xi_{\left(A_{1}\right.}^{\dot{Z}_{1} \dot{z}_{2}} \nabla_{\left.A_{2}\right)}^{\dot{z}_{3}} \bar{\Psi}_{\dot{X} \dot{z}_{1} \dot{z}_{2} \dot{z}_{3}} \\
& +\frac{2}{3}\left(\Phi_{A_{1} A_{2} \dot{X} z_{1}} \nabla_{C Z_{2}} \xi^{C \dot{z}_{1} \dot{z}_{2}}-\Phi_{z_{1} z_{2} C\left(A_{1}\right.} \nabla_{\left.A_{2}\right) \dot{X}} \xi^{C \dot{z}_{1} \dot{z}_{2}}\right) \\
& +\frac{1}{3} \xi^{C Z_{1} z_{2}} \nabla_{\left(A_{1} \dot{X}\right.} \Phi_{\left.A_{2}\right) C Z_{1} z_{2}}+2 \xi_{\left(A_{1} \dot{x}\right.} \dot{z}_{\left.A_{2}\right) \dot{Z}} \Lambda .
\end{aligned}
$$

This difference shows that one can generalize Lemma 2 for $n=2$ at 3 Analysis, Bd. 11, Heft 1 (1992) 
most to spaces of constant curvature. Summarizing, it seems to be impossible to generalize the results of Section 2 for $n>1$ to a curved spacetime unless the latter one is of constant curvature.

One possibility to overcome this situation is to consider the field equations

$$
\left.\nabla_{\left(\dot{X}^{\prime}\right.}^{A} \xi_{i}: \dot{x}_{1} \ldots \dot{x}_{n}\right)=0, \quad \xi \in \hat{S}_{1, n}
$$

which are obviously stronger than (21). Equation (27) is a suitable candidate to describe (possibly a special class of) massless fields with arbitrary spin in arbitrarily curved spacetimes, as the following theorem shows.

Theorem 1: The equations (27) have the following properties:

i) The equations (27) are consistent on a generally curved spacetime for all $n \geq 0$. For these equations the Cauchy problem is properly posed.

ii) In flat spacetimes the solutions of (27) satisfy the equation (21) for the potential $\xi$ of the field $\varphi$.

iii) The equations (27) are conformally invariant.

iv) If $\varpi \in \hat{S}_{0, n-1}$ is some given spinor field, then there exists a solution $\xi$ of (27) such that

$$
\nabla^{A \dot{X}_{1}} \xi_{A \dot{X}_{1} \dot{x}_{2}} \ldots \dot{x}_{n}=\tilde{\omega}_{\dot{X}_{2} \ldots \dot{x}_{n}}
$$

Proof: The Cauchy problem for the system (27), (28) was studied in $[10,15]$. Existence and uniqueness of its solution are proved in these papers. The conformal invariance of (27) is shown in [13]. The statement ii) is obviously correct

Remark 3: Suggested by Theorem 1 one can ask whether one can generalize equations (27) to $\xi \in \hat{S}_{k, n}$ for $k>1$ since it is known that the arising equations are conformally invariant, too [13]. But the arising equations are inconsistent with the exeption of the following cases $[3,10,15]$ :

$$
\begin{array}{ll}
k=2, n=0 & k>2, n=0 \text { and } \Phi=0 \\
k=2, n>0 \text { and } \Phi=0 & k>2, n>0 \text { and } \Phi=\Psi=0 .
\end{array}
$$


Remark 4: The second order equations

$$
\left.\nabla_{B}^{\dot{x}} \nabla_{(\dot{X}}^{A} \xi_{i A i \dot{x}_{1}} \ldots \dot{x}_{n}\right)=0, \quad \xi \in \hat{S}_{1, n}
$$

are conformally invariant only in the case of $n=1$, i.e. for Maxwell fields (see Proposition 4 and Remark 2).

\section{REFERENCES}

[1] BARTH, N. H., and S. M. ChRISTENSEN: Arbitrary spin field equations on curved manifolds with torsion. J. Phys. A 16 (1983), 543-563.

[2] BFNGTSSON, A. K. H., and J. BENGTSSON: Massless higher-spin fields revisited. Class. Quantum Grav. 3 (1986), 927-936.

[3] BUCHDAHI., H. A.: On the compatibility of relativistic wave equations in Riemann spaces. Nuovo Cimento 25 (1962), $486-496$.

(4) Coll.iNs, G. P., and N. A. DoughtY: Systematics of arbitrary-helicity Lagrangian wave equations. J. Math. Phys. 28 (1987), 448-456.

[5] DiRac, P. A. M.: Relativistic wave equations. Proc. Roy. Soc. A 155 (1936), 447-459.

[6] FANG, J., and C. FRONSDAL: Massless fields with half-integer spin. Phys. Rev. D 18 (1978), $3630-3633$.

[7] FIERZ, M.: Über die relativistische Theorie kräftefreier Teilchen mit beliebigem Spin. Helv. Phys. Acta 12 (1939), 3-37.

[8] FIER7, M., and W. PALLI: On relativistic wave equations for particles of arbitrary spin in an electromagnetic field. Proc. Roy. Soc. A 173 (1939), 211-232.

[9] Fronsdal,C.: Massless fields with integer spin. Phys. Rev. D 18 (1978), 3624-3629.

[10] II.1.GF, R.: On potentials for several classes of spinor and tensor fields in curved spacetimes. Gen. Rel. Grav. 20 (1988), 551 - 564.

[11] VAN Nifumlenhlitzen, P.: Supergravity. Phys. Rep. 68 (1981), 189-398.

[12] PLenrose, R.: Zero rest-mass fields including gravitation: asymptotic behavior. Proc. Roy. Soc. A 284 (1965), $159-203$.

[13] Penrose, R., and W. Rindler: Spinors and Space-Time. Parts I and II. Cambridge: Cambridge University Press 1984 and 1986.

[14] PFinose, R., and R. S. WARD: Twistors for flat and curved spacetime. In: Gencral Relativity and Gravitation, Vol 2 (Ed.: A. Held). New York and London: Plenum Press 1980, pp. 283-315.

[15] WüssCII, V.: Cauchy-Problem und Huygenssches Prinzip bei einigen Klassen spinorieller Feldgleichungen. Beitr. Anal. 12 (1978), 47-76.

Received 22. 11. 1990; in revised form 24.07. 1991

Dr. sc. Reinhard Illge

Fachbereich Mathematik der Pädagogischen Hochschule

Nordhäuser Straße 63

D(Ost) - 5064 Erfurt 